\title{
Reduced Fhit expression is associated with mismatch repair deficiency in human advanced colorectal carcinoma
}

\author{
H Andachi', K Yashima*,', M Koda', K Kawaguchi', A Kitamura', A Hosoda', Y Kishimoto², G Shiota', H Ito ${ }^{3}$, \\ M Makino ${ }^{4}$, N Kaibara ${ }^{4}$, H Kawasaki' and Y Murawaki'
}

'Second Department of Internal Medicine, Faculty of Medicine, Tottori University, Yonago 683-8504, Japan; ${ }^{2}$ Department of Clinical Pharmacology, Faculty of Medicine, Tottori University, Yonago 683-8504, Japan; ${ }^{3}$ First Department of Pathology, Faculty of Medicine, Tottori University, Yonago 683-8504, Japan;

${ }^{4}$ First Department of Surgery, Faculty of Medicine, Tottori University, Yonago 683-8504, Japan

The Fragile Histidine Triad gene, encompassing the FRA3B fragile site at chromosome 3p I4.2, is a candidate tumour suppressor gene involved in multiple tumour types including colorectal carcinomas. Recently, it has been reported that the Fragile Histidine Triad gene may be a target of damage in a fraction of mismatch deficient tumours. To explore this hypothesis, we analysed both Fragile histidine triad and mismatch repair protein (Msh2 and MlhI) expression using immumohistochemical methods in 52 advanced colorectal carcinomas ( 19 well-, 17 moderately-, and 16 poorly-differentiated). In addition, we examined whether the Fragile histidine triad and mismatch repair protein expression correlated with p53 expression and clinicopathological findings. Significant loss or reduction of Fragile histidine triad expression was noted in 18 of the 52 (34.6\%) advanced colorectal carcinomas: 2 (I0.5\%) well-differentiated, 3 (17.6\%) moderately-differentiated, 13 (81.3\%) poorly-differentiated carcinomas, the frequency being significantly higher in the latter than that in the former two $(P<0.000 \mathrm{I})$. Loss of mismatch repair protein (mainly, MIhI) expression was detected in 21 of the 52 (40.4\%) colorectal carcinomas. Moreover, reduced Fragile histidine triad expression was significantly associated with absence of mismatch repair protein expression in the advanced colorectal carcinomas $(P<0.000 \mathrm{I})$. However, the Fragile histidine triad and mismatch repair protein expression was not significantly associated with p53 expression. These results suggested that reduced Fragile histidine triad expression might be correlated with mismatch repair expression, but not with $\mathrm{p} 53$ expression.

British Journal of Cancer (2002) 87, 44I-445. doi:I0.1038/sj.bjc.660050I www.bjcancer.com

(c) 2002 Cancer Research UK

Keywords: colorectal carcinoma; immunohistochemistry; Fhit; Msh2; Mlhl; p53

A candidate tumour suppressor gene, Fragile Histidine Triad (FHIT), was identified at chromosome 3p14.2 spanning the FRA3B common fragile site (Ohta et al, 1996). The FHIT gene consists of 10 exons, which distribute over a genomic region of $>1 \mathrm{Mb}$. The Fragile histidine triad (Fhit) protein has been characterised as an $\mathrm{Ap}_{3} \mathrm{~A}$ hydrolase molecule, which cleaves the $\mathrm{Ap}_{4} \mathrm{~A}$ substrate that may be involved in the control of DNA replication and the cell cycle (Ohta et al, 1996). Frequent abnormal transcripts were found in a variety of human cancers including those of the digestive tract, lung, breast, and head and neck (Ohta et al, 1996; Croce et al, 1999; Kitamura et al, 2001). The majority of these abnormalities include aberrant mRNA transcripts, with the absence of one or more exons within the mRNA. Genomic analysis demonstrated frequent allelic loss and homozygous deletions (Ohta et al, 1996; Croce et al, 1999).

In CRCs, Ohta et al (1996) reported that aberrant transcripts of the FHIT gene were detected in three of eight tumours by nested RT-PCR. In contrast, Thiagalingam et al (1996) reported that aberrant transcripts were rare using RT-PCR, suggesting that FHIT is inactivated by an unual mechanism or that it plays a role in relatively few colorectal tumours. Other reports suggested

*Correspondence: Kazuo Yashima;

E-mail: yashima@grape.med.tottori-u.ac.jp.

Received I 4 January 2002; revised 9 May 2002; accepted I0 June 2002 that Fhit does indeed play a role in the development and progression of colorectal carcinomas (CRCs) (Hibi et al, 1997; Hao et al, 2000; Luceri et al, 2000; Mori et al, 2001).

Defects in the DNA mismatch repair (MMR) system are involved in the carcinogenesis and tumour progression of sporadic and inherited human cancers (Eshleman et al, 1996; Kinzler and Vogelstein, 1996). MMR deficiency leads to the accumulation of base-base mismatches and short insertion/deletion mispairs, generated as a consequence of DNA replication errors and homologous recombinations. Inherited mutations of the $\mathrm{MLH1}$ and $\mathrm{MSH} 2$ genes have been demonstrated as the cause of more than $90 \%$ of hereditary non-polyposis colorectal cancers (HNPCC) (Peltomaki and Vasen, 1997). MMR genes are also involved in the development of a subset of sporadic colorectal, gastric and endometrial tumours (Duggan et al, 1994; Halling et al, 1999; Ward et al, 2001). MMR deficiency, identified by the presence of microsatellite instability (MSI), occurs in approximately $10-15 \%$ of sporadic CRCs (Kinzler and Vogelstein, 1996). Most sporadic CRCs with MSI have been demonstrated to be caused by somatic hypermethylation of the MLH1 promoter region, resulting in the downregulation of $M L H 1$ gene expression (Herman et al, 1998). Recent data have revealed that immunohistochemistry is an accurate screening technique to identify MMR deficient tumours (Thibodeau et al, 1996; Marcus et al, 1999).

Recently, Fong et al (2000) have demonstrated that NMBA (Nnitrosomethylbenzylamine) exposure caused a spectrum of visceral 
and skin tumours similar to Muir-Torre syndrome, caused by a deficiency in a MMR gene, in Fhit-deficient mice, and suggested that the FHIT gene may be a target of damage in a fraction of mismatch deficient tumours. Moreover, Mori et al (2001) reported that the loss of Msh2 protein is significantly correlated with the loss of Fhit expression in human CRCs. However, the relationship between Fhit and the MMR gene (both Msh2 and Mlh1) expression has not been previously studied in detail using clinical samples.

In this study, we examined the immunohistochemical expression of Fhit, Msh2, Mlh1, and p53 in advanced CRCs to explore the hypothesis that the Fhit inactivation is a frequent result of MMR deficiency.

\section{MATERIALS AND METHODS}

\section{Patient samples}

Tumour blocks were obtained from 52 patients, who underwent colorectomy at Tottori University Hospital between 1992 and 1996. Patients with familial adenomatous polyposis (FAP) were excluded, but family history was not an exclusion criterion; therefore, some cases of HNPCC may have been included in the study population. The ages of the 27 male and 25 female patients ranged from 51 to 90 years (mean \pm SD: $67.4 \pm 1.32$ years). Pathologically, all of the tumours were advanced adenocarcinomas (19 well-differentiated, 17 moderately-differentiated, and 16 poorlydifferentiated). Nineteen cases had right sided tumours and 33 cases had left sided tumours. Right-sided lesions were defined as those confined to the coecum or ascending or transverse colon, whereas left-sided lesions were defined as being confined to the descending colon, sigmoid colon, or rectum. On the basis of Dukes' classification, four were in stage A, 12 in stage B, 21 in stage $\mathrm{C}$, and 15 in stage D. Lymph node metastases were present in 34 of the $52(59.6 \%)$ patients. Histological classification was made according to the criteria of the Colorectal Cancer Study Group of Japan (Colorectal Cancer Study Group of Japan, 1998). All diagnoses of pathological specimens were verified by two experienced pathologists (H.A. and H.I.). All the cases were analysed anonymously, i.e., all the specimens were given new numbers without names. Institutional Review Board approval was obtained.

\section{Immunohistochemical staining}

Paraffin-embedded, $4 \mu \mathrm{m}$-thick sections were immunohistochemically stained with anti-FHIT rabbit polyclonal antibody (IBL, Gunma, Japan; dilution 1:100), anti-MSH2 mouse monoclonal antibody (FE11, Oncogene Research Products, Cambridge, MA, USA); dilution 1:100), anti-MLH1 mouse monoclonal antibody (G168-15, PharMingen, San Diego, CA, USA; dilution 1:50), and anti-p53 mouse monoclonal antibody (DO-7, Dakopatts, Copenhagen, Denmark; dilution 1:50) using the avidin-biotin-peroxidase complex technique. Immunohistochemical staining was performed as described below. In brief, after deparaffinising in xylene and dehydrating in ethanol, the sections were immersed in a citrate buffer $(0.01 \mathrm{M}, \mathrm{pH} 6.0)$ and heated in a microwave oven for $15 \mathrm{~min}$ to retrieve antigens, then incubated with the primary antibody overnight at $4{ }^{\circ} \mathrm{C}$. As a negative control, the primary antibody was replaced with normal serum IgG in a similar dilution. The detection reaction followed the Vectastain Elite $\mathrm{ABC}$ kit protocol (Vector Laboratories, Burlingame, CA, USA). Diaminobenzidine was used as a chromogen, and methylgreen or haematoxylin was used as a counterstain. The sections were then incubated with biotinylated anti-rabbit IgG and avidin-biotin-peroxidase and visualised using diaminobenzidine tetrahydrochloride. The immunohistochemical expressions were evaluated by two independent observers (H.A. and K.Y.). Immunohistochemical analysis was performed in a blinded manner with respect to the clinical information.

\section{Assessment of Fhit immunostaining}

The Fhit expression was graded for both the extent and the intensity of immunopositivity as described previously Hao et al (2000). The extent of positivity was scored as follows: $0,<5 \% ; 1,>5-25 \% ; 3$, $>50-75 \%$; and $4,>75 \%$ of the colonic epithelial cells in the respective lesions. The intensity was scored as follows: 0 , negative; $1+$, weak; $2+$, moderate; and $3+$, as strong as normal mucosa. The final score was obtained by multiplying the extent of positivity and intensity scores, producing a range from 0 to 12 . Scores $9-12$ were defined as preserved or strong staining pattern, scores $0-4$ were defined as markedly reduced or lost expression, and scores $5-8$ were defined as intermediate staining pattern.

\section{Assessment of Msh2 and Mlh1 immunostaining}

Normal tissue adjacent to the tumour was used as an internal positive control. The normal staining pattern for both hMlh1 and hMsh2 was nuclear. Tumour cells that exhibited an absence of nuclear staining in the presence of non-neoplastic cells with nuclear staining were considered to have an abnormal pattern.

\section{Assessment of p53 immunostaining}

Five representative fields were examined, and a total of 1000 tumour cells (200 for each field) were counted under the microscope with a high power $(\times 200)$ objective. A distinct nuclear immunoreaction was assessed as positive. In this study, the specimens were regarded as p53-positive when over $25 \%$ of the tumour cells showed positive signals.

\section{Statistical evaluation}

Statistical analysis was performed by the Fisher's exact test. $P<0.05$ was considered significant.

\section{RESULTS}

\section{Fhit expression in the normal mucosa and advanced cancers}

By immunohistochemical staining, all the normal colonic epithelia showed strong cytoplasmic expression of the Fhit protein from the basal cells to the luminal differentiated cells (Figure 1A); these served as internal controls. Smooth muscle cells and inflammatory mononuclear cells were positive at various intensities and to various degrees.

Reduced or absent Fhit expression was noted in 18 of the 52 (34.6\%) advanced CRC cases, preserved in 25 (48.1\%) CRCs and intermediate in nine $(17.3 \%)$ CRCs (Table 1, Figure 1A,B). The intensity of the staining of the colorectal carcinoma cells was determined by comparing it to that of the normal colonic epithelial cells found within the same specimen (Figure 1A).

We analysed the relationship between these results and the clinicopathological data (tumour location, histological differentiation, Dukes' stage, lymph node metastasis, and p53 expression). Decreased staining or lack of staining for Fhit was detected in 2 of $19(10.5 \%)$ well-differentiated cancers, in three of $17(17.6 \%)$ moderately-differentiated cancers, and in the 13 of $16(81.3 \%)$ poorly-differentiated cancers. The incidence of reduced Fhit expression in CRCs was significantly highest in the poorly-differentiated histology $(P<0.0001$; Table 1$)$. However, no significant associations were found among the Fhit expression and other clinicopathological parameters. 
Table I Relationship between Fhit and MMR protein expression and clinicopathological findings in advanced colorectal carcinoma

\begin{tabular}{|c|c|c|c|c|c|c|c|}
\hline & \multirow[b]{2}{*}{$n$} & \multicolumn{3}{|c|}{ Fhit protein expression } & \multicolumn{3}{|c|}{$\begin{array}{l}\text { MIhI/Msh2 } \\
\text { expression }\end{array}$} \\
\hline & & $\begin{array}{l}\text { preserved/ } \\
\text { intermediate }\end{array}$ & $\begin{array}{l}\text { reduced or } \\
\text { absent }\end{array}$ & $\mathbf{P}$ & + & - & $\mathbf{P}$ \\
\hline \multicolumn{8}{|c|}{ Histological type } \\
\hline well & 19 & 17 & $2 \longrightarrow$ & \multirow{3}{*}{$<0.0001$} & 13 & $6 \longrightarrow$ & \multirow{3}{*}{0.0128} \\
\hline moderately & 17 & 14 & 3 & & 13 & 4 & \\
\hline poorly & 16 & 3 & 13 & & 5 & 11 & \\
\hline \multicolumn{8}{|c|}{ Tumour localization } \\
\hline right side & 19 & 11 & 8 & \multirow[t]{2}{*}{ NS } & 7 & 12 & \multirow[t]{2}{*}{0.0184} \\
\hline left side & 33 & 23 & 10 & & 24 & 9 & \\
\hline \multicolumn{8}{|l|}{ Dukes' stage } \\
\hline A & 4 & 4 & 0 & \multirow[t]{4}{*}{ NS } & 3 & 1 & \multirow[t]{4}{*}{ NS } \\
\hline B & 12 & 8 & 4 & & 6 & 6 & \\
\hline C & 21 & 10 & II & & 10 & 11 & \\
\hline D & 15 & 12 & 3 & & 12 & 3 & \\
\hline \multicolumn{8}{|c|}{ Lymph node metastasis } \\
\hline+ & 34 & 20 & 14 & \multirow[t]{2}{*}{ NS } & 20 & 14 & \multirow[t]{2}{*}{ NS } \\
\hline- & 18 & 14 & 4 & & 11 & 7 & \\
\hline \multicolumn{8}{|l|}{ p53 expression } \\
\hline+ & 35 & 24 & 11 & \multirow{2}{*}{ NS } & 22 & 13 & \multirow[t]{2}{*}{ NS } \\
\hline- & 17 & 10 & 7 & & 9 & 8 & \\
\hline
\end{tabular}

Right-sided lesions were defined as those proximal to the splenic flexure. NS: not significant.
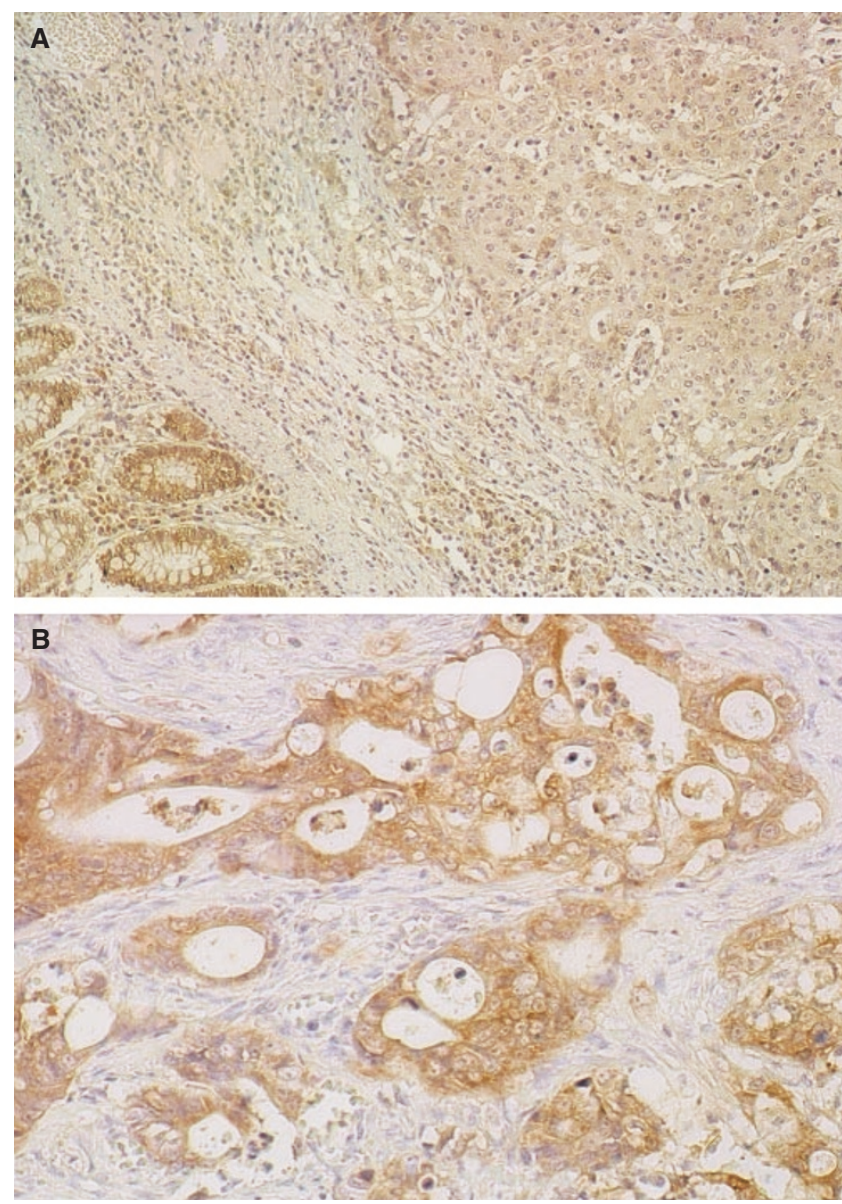

Figure I Representative results of Fhit immunostaining in human normal and carcinomatous colorectal tissues. (A), Reduced immunostaining of a tumour and positive immunostaining of normal colonic epithelium; (B), Positive immunostaining of an invasive tumour

\section{Correlation of Fhit expression with MMR gene expression}

The expression of both Mlh1 and Msh2 proteins was without exception nuclear. In the normal mucosa, they were detected predominantly in the areas of active proliferation, such as the germinal centres of the lymphoid follicles and the lower portions of the normal colonic crypts (Figure 2A,C). Normal stromal cells such as fibroblasts and endothelial cells also showed nuclear positivity for both the proteins. We demonstrated that 19 of the $52(36.5 \%)$ tumours had reduced expression levels of the Mlh1 protein (Figure $2 \mathrm{~B})$, whereas five $(9.6 \%)$ carcinomas had reduced expression levels of the Msh2 protein (Figure 2D). Reduced expression levels of both proteins were observed in three of the $52(5.8 \%)$ specimens (Table 2). In addition, loss of nuclear Mlh1 or Msh2 expression was more frequently associated with poor differentiation $(P=0.0128)$ and right-sided location $(P=0.0184)$ (Table 1$)$. However, there was no significant difference in other clinicopathological parameters (age, gender, Dukes stage, node metastasis, and p53 expression). Among the tumours with reduced or absent Fhit expression, $72.2 \%$ (14 of 18) had loss of nuclear Mlh1 or Msh2 expression compared with only $20.6 \%$ (seven of 34 ) of the preserved or intermediate Fhit expression tumours $(P<0.0001)$ (Table 2$)$.

\section{DISCUSSION}

Alterations and abnormal transcripts of the FHIT gene have been reported in a number of primary human tumours, including CRCs (Ohta et al, 1996; Croce et al, 1999; Kitamura et al, 2001). However, some of the data are conflicting in CRCs (Hao et al, 2000; Hibi et al, 1997; Luceri et al, 2000; Mori et al, 2001; Thiagalingam et al, 1996). Recently, it was reported that alterations in the FHIT locus detected by DNA and/or reverse transcription-PCR analysis correlated with a loss of Fhit protein expression in lung, cervical, and oesophageal carcinomas (Birrer et al, 1999; Mori et al, 2000; Sozzi et al, 1998). These results indicated that FHIT gene alteration can be simply detected by immunohistochemical analysis of tumour specimens. In this study, we observed frequent abnormal Fhit protein expression in poorly-differentiated CRCs, with $77 \%$ of the specimens demonstrating a decrease in, or lack of, Fhit 

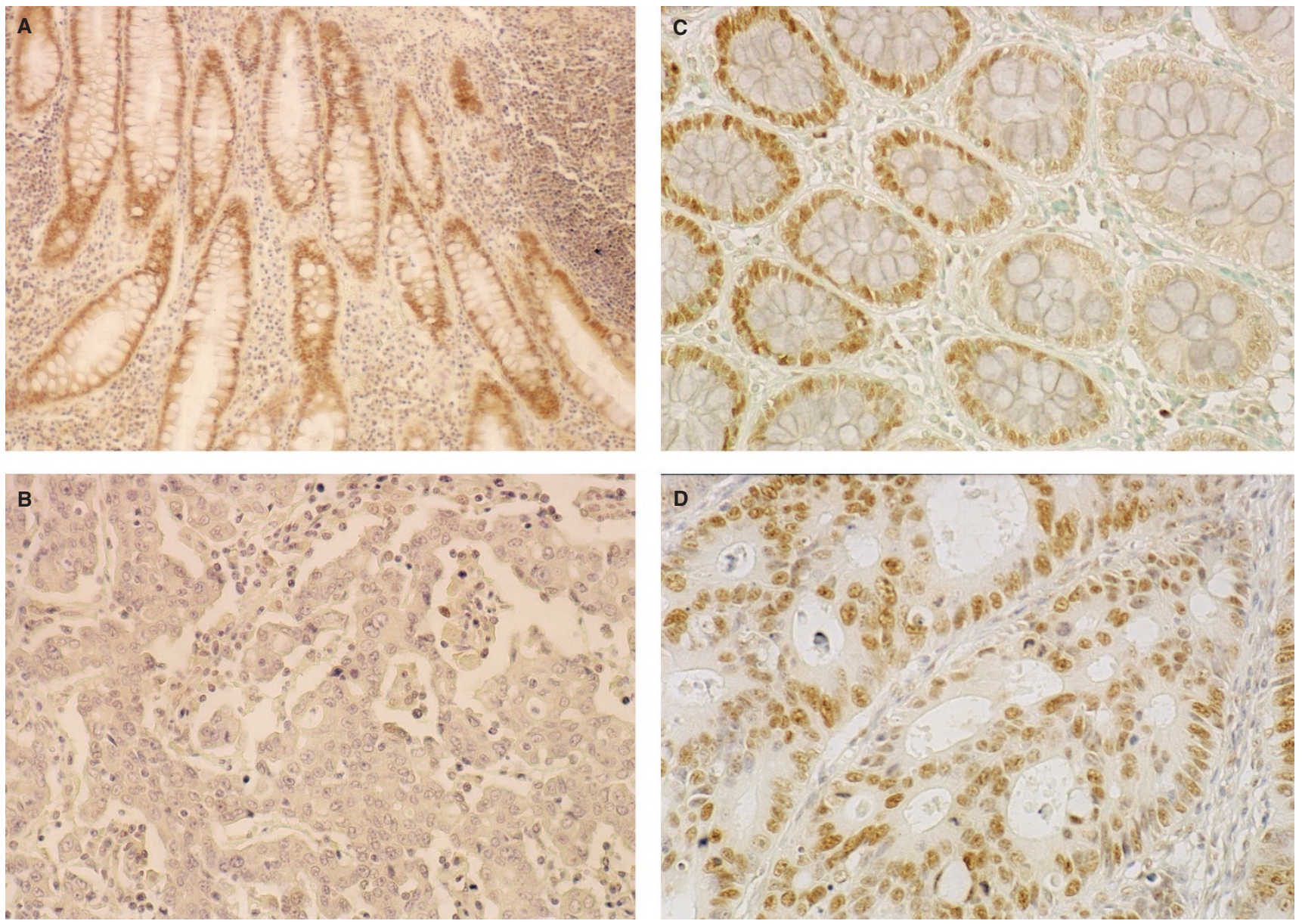

Figure 2 Representative results of MlhI and Msh2 immunostaining in human normal and carcinomatous colorectal tissues. A and B, Poorly-differentiated carcinoma cells (B) show no nuclear staining for Mlh I while normal crypt epithelium $(\mathbf{A})$ shows nuclear staining; (C) Normal crypt epithlium shows nuclear staining for Msh2; (D), Moderately-differentiated carcinoma cells show nuclear staining for Msh2

Table 2 Relationship between Fhit and MMR protein expression in colorectal carcinoma

\begin{tabular}{lccc}
\hline & & \multicolumn{2}{c}{ Fhit expression } \\
\cline { 3 - 4 } & $\boldsymbol{n}$ & $\begin{array}{c}\text { Preserved/ } \\
\text { intermediate }\end{array}$ & $\begin{array}{c}\text { Reduced } \\
\text { or absent }\end{array}$ \\
\hline MIhI (+), Msh2 (+) & 31 & 27 & 4 \\
MIhI (-), Msh2 (+) & 16 & 4 & 12 \\
MIhI (+), Msh2 $(-)$ & 2 & 1 & 1 \\
MIhI (-), Msh2 (-) & 3 & 2 & 1 \\
\hline
\end{tabular}

Reduced Fhit expression is associated with loss of MMR expression: $P<0.0001$.

protein staining. This frequency of abnormal Fhit expression was similar to that observed in poorly-differentiated CRCs by Hao et al (2000). Therefore, these results suggest that abnormal Fhit expression is associated with decreasing degrees of differentiation in CRCs. However, we found no correlation between the Fhit expression and any of the clincopathological parameters including p53 expression.

Inactivation of MMR genes is a recently described alternate pathway in cancer development and progression (Eshleman and Makowitz, 1996; Kinzler and Vogelstein, 1996). MMR deficiency is present in $10-15 \%$ of sporadic CRCs (Kinzler and Vogelstein,
1996), and is the underlying cause of more than $90 \%$ of HNPCC (Peltomaki and Vasen, 1997). Previous studies demonstrated that immunohistochemical analysis of the expression of Mlh1 and Msh2 could be an accurate and rapid screening procedure for the identification of MMR gene alterations (Thibodeau et al, 1996; Marcus et al, 1999). We found abnormal Mlh1 or Msh2 expression in 21 of the 52 (46\%) CRCs using immunohistochemical methods. This frequency of abnormal MMR protein expression was more frequent than that observed in CRC by other investigators (Kinzler and Vogelstein, 1996). In the assessment of MMR protein expression, we used almost the same criteria used by other groups (Marcus et al, 1999; Ward et al, 2001). In addition, it was reported that sporadic CRCs with MSI were more frequent in the poorly differentiated phenotype (Ward et al, 2001). In our study, the ratio of poorly differentiated CRCs was high compared with previous reports (Kinzler and Vogelstein, 1996; Marcus et al, 1999; Ward et al, 2001). Therefore, high frequency of abnormal MMR protein expression might be mainly due to the different distributions of histological differentiation. Moreover, we found a significant correlation between the MMR protein expression and histological differentiation and tumour location. These results were also consistent with previous reports (Kinzler and Vogelstein, 1996). In the present study, MMR deficiency was mainly due to the loss of Mlh1 expression, suggesting that MLH1 hypermethylation is the predominant mechanism (Herman et al, 1998). 
Recently, it has been reported that by NMBA (N-nitrosomethylbenzylamine) exposure, Fhit-deficient mice developed a spectrum of visceral and skin tumours similar to Muir-Torre syndrome, caused by a deficiency in a MMR gene (Fong et al, 2000). A large subgroup of MTS cases exhibits MSI and germline mutations in the MLH1 or MSH2 gene (Kruse et al, 1998). In addition, it was previously observed that human pancreatic cancers and cell lines with high MSI frequently had homozygous deletions within FHIT (Hilgers and Kern, 1999; Hilgers et al, 2000). Therefore, these reports suggested that the FHIT gene might be a target of damage in a fraction of mismatch deficient tumours. Our data show that MMR deficiency based on the status of the Mlh1 and Mlh2 protein expression is significantly associated with reduced Fhit expression in advanced colorectal carcinomas and support this hypothesis. Mori et al (2001) reported an association between Msh2 absence and FHIT altera-

\section{REFERENCES}

Birrer MJ, Hendricks D, Farley J, Sundborg MJ, Bonome T, Walts MJ, Geradts J (1999) Abnormal Fhit expression in malignant and premalignant lesions of the cervix. Cancer Res 59: 5270-5274

Croce CM, Sozzi G, Huebner K (1999) Role of Fhit in human cancer. J Clin Oncol 17: $1618-1624$

Duggan BD, Felix JC, Muderspach LI, Tourgeman D, Zheng J, Shibata D (1994) Microsatellite instability in sporadic endometrial carcinoma. J Natl Cancer Inst 86: 1216-1221

Eshleman JR, Makowitz SD (1996) Mismatch repair defects in human carcinogenesis. Hum Mol Genet 5: $1489-1494$

Fong LYY, Fidanza V, Zanesi N, Lock LF, Siracusa LD, Mancini R, Siprashvili Z, Ottey M, Martin SE, Druck T, McCue PA, Croce CM, Heubner K (2000) Muir-Torre-like syndrome in Fhit-deficient mice. Proc Natl Acad Sci USA 97: $4742-4747$

Halling KC, Harper J, Moskaluk CA, Thibodeau SN, Petroni GR, Yustein AS, Tosi P, Minacci C, Roviello F, Piva P, Hamilton SR, Jackson CE, Powell SM (1999) Origin of microsatellite instability in gastric cancer. Am J Pathol 155: $205-211$

Hao XP, Willis JE, Pretlow TG, Rao JS, MacLnnan GT, Talbot IC, Pretlow TP (2000) Loss of fragile histidine triad expression in colorectal carcinomas and premalignant lesions. Cancer Res 60: 18-21

Herman JG, Umar A, Polyak K, Graft JR, Ahuja N, Issa JPJ, Markowitz S, Wilson JKV, Hamilton SR, Kinzler KW, Kane MF, Kolodner RD, Vogelstein B, Kunkel TA, Baylin SB (1998) Incidence and functional consequences of hMLH1 promoter hypermethylation in colorectal carcinoma. Proc Natl Acad Sci USA 95: 6870-6875

Hibi K, Taguchi M, Nakamura H, Hirai A, Fujikake Y, Matsui T, Kasai Y, Akiyama S, Ito K, Takagi H (1997) Altenative splicing of the FHIT gene in colorectal cancers. Jpn J Cancer Res 88: 385-388

Hilgers W, Kern SE (1999) Molecular genetic basis of pancreatic adenocarcinoma. Genes Chromosomes Cancer 26: 1-12

Hilgers W, Koerkamp BG, Geradts J, Tang DJ, Yeo CJ, Hruban RH, Kern SE (2000) Genomic FHIT analysis in RER+ and RER- adenocarcinomas of the pancreas. Genes Chromosomes Cancer 27: 239-243

Colorectal Cancer Study Group of Japan (1998) The general rules for the clinical and pathological study of primary colorectal cancer, ed 6 Tokyo: Kinbara

Kinzler KW, Vogelstein B (1996) Lessons from hereditary colorectal cancer. Cell 87: $159-170$

Kitamura A, Yashima K, Okamoto E, Andachi H, Hosoda A, Kishimoto Y, Shiota G, Ito H, Kaibara N, Kawasaki H (2001) Reduced Fhit expression occurs in the early stage of esophageal tumorigenesis: no correlation with p53 expression and apoptosis. Oncology 61: $205-211$

Kruse R, Rutten A, Lamberti C, Hosseiny-Malayeri HR, Wang Y, Ruelfs C, Jungck M, Mathiak M, Ruzicka T, Hartschuh W, Bisceglia M, Friedl W, Propping P (1998) Muir-Torre phenotype has a frequency of DNA mismatch-repair- gene mutations similar to that in hereditary nonpolyposis colorectal cancer families defined by the Amsterdam criteria. Am J Hum Genet 63: $63-70$ tions in CRCs but did not analyse Mlh1 expression. Moreover, as the mechanisms of this hypothesis, they proposed that the repetitive elements, such as (CA)n and (A)n repeats, in introns 4 and 5 of the FHIT gene could be a target of damage in MMR deficient tumours.

Thus, we noted an association between Fhit and MMR protein expression, but it could be due to the result of an association between Fhit and MMR protein expression and poorly differentiation.

In conclusion, we have demonstrated that reduced Fhit expression was associated with the loss of MMR protein expression in advanced CRCs. In combination with previous reports, this result supports the possibility that FHIT gene alterations could be caused by a deficiency in a MMR gene. Studies that look into the regulation of Fhit and MMR protein expression in cancer may offer a new insight into colorectal carcinogenesis and plausibly, chemopreventive pathways.
Luceri C, Guglielmi F, De Filippo C, Canderni G, Mini E, Biggeri A, Napoli C, Tonelli F, Cianchi F, Dolara P (2000) Clinicopathologic features and FHIT gene expression in sporadic colorectal adenocarcinomas. Scand J Gastroenterol 35: 637-641

Marcus VA, Madlensky L, Gryfe R, Kim H, So K, Millar A, Temple LKF, Hsieh E, Hiruki T, Narod S, Bapat BV, Gallinger S, Redston M (1999) Immunohistochemistry for hMLH1 and hMSH2: a practical test for DNA mismatch repair-deficient tumors. Am J Surg Pathol 23: 1248-1255

Mori M, Mimori K, Shiraishi T, Alder H, Inoue H, Tanaka Y, Sugimachi K, Huebner K, Croce CM (2000) Altered expression of Fhit in carcinoma and precarcinomatous lesions of the esophagus. Cancer Res 60: 1177-1182

Mori M, Mimori K, Masuda T, Yoshinaga K, Yamashita K, Matsuyama A, Inoue $H$ (2001) Absence of Msh2 protein expression is associated with alteration in the FHIT locus and Fhit expression in colorectal carcinoma. Cancer Res 61: 7379-7382

Ohta M, Inoue H, Cotticelli MG, Kastury K, Baffa R, Palazzo J, Siprashvili Z, Mori M, Macue P, Druck T, Croce CM, Huebner K (1996) The FHIT gene, spanning the chromosome 3p14.2 fragile site and renal carcinoma-associated t $(3 ; 8)$ breakpoint, is abnormal in digestive tract cancers. Cell 84: $587-597$

Peltomaki P, Vasen HF (1997) Mutations predisposing to hereditary nonpolyposis colorectal cancer: database and results of a collaborative study. The International Collaborative Group on Hereditary Nonpolyposis Colorectal Cancer. Gastroenterology 113: 1146-1158

Sozzi G, Pastorino U, Moiraghi L, Tagliabue E, Pezzella F, Ghirelli C, Tornielli S, Sard L, Huebner K, Pierotti MA, Croce CM, Pilotti S (1998) Loss of Fhit function in lung cancer and preinvasive bronchial lesion. Cancer Res 58: $5032-5037$

Thiagalingam S, Lisitsyn NA, Hamaguchi M, Wigler MH, Willson JKV, Markowitz SD, Leach FS, Kinzler KW, Vogelstein B (1996) Evaluation of the FHIT gene in colorectal cancers. Cancer Res 56: 2936-2939

Thibodeau SN, French AJ, Roche PC, Cunningham JM, Tester DJ, Lindor NM, Moslein G, Baker SM, Liskay RM, Burgart LJ, Honchel R, Halling KC (1996) Altered expression of hMSH2 and hMLH1 in tumors with microsatellite instability and genetic alterations in mismatch repair gene. Cancer Res 56: $4836-4840$

Ward R, Meagher A, Tomlinson I, O'Connor T, Norrie M, Wu R, Hawkins N (2001) Microsatellite instability and the clinicopathological features of sporadic colorectal cancer. Gut 48: 821-829 